OPEN ACCESS

Edited by:

Hassane Zouhal,

University of Rennes 2 - Upper

Brittany, France

Reviewed by:

Marc Vanderthommen,

University of Liège, Belgium

Brian H. Dalton,

University of British Columbia

Okanagan, Canada

${ }^{*}$ Correspondence:

Thierry Paillard

thierry.paillard@univ-pau.fr

Specialty section:

This article was submitted to

Exercise Physiology,

a section of the journal

Frontiers in Physiology

Received: 18 September 2021

Accepted: 07 December 2021

Published: 12 January 2022

Citation:

Paillard T (2022) Neuromuscular or Sensory Electrical Stimulation for Reconditioning Motor Output and

Postural Balance in Older Subjects?

Front. Physiol. 12:779249.

doi: 10.3389/fphys.2021.779249

\section{Neuromuscular or Sensory Electrical Stimulation for Reconditioning Motor Output and Postural Balance in Older Subjects?}

\author{
Thierry Paillard *
}

Laboratoire Mouvement, Equilibre, Performance et Santé, EA 4445, Département STAPS, Université de Pau et des Pays de l'Adour, E2S, Tarbes, France

Percutaneous electrical stimulation is used for reconditioning functional capabilities in older subjects. However, its optimal application depends on the specific physiological needs of the individual. Depending on whether his/her needs are related to motor function or sensory and central functions, the relevant modality of electrical stimulation differs significantly. In fact, there are two main modalities of electrical stimulation, that is, neuromuscular electrical stimulation (NMES) and sensory electrical stimulation (SES). NMES involves high-intensity currents (above the motor threshold) and provokes involuntary visible direct muscle contractions. With chronic application, the induced adaptations occur mainly at the neuromuscular function level and thus enhance muscle strength/power and motor output. SES involves low-intensity currents (below, at or only just above the sensory threshold), does not induce any visible muscle contraction and provides only sensory information. With chronic application, the induced adaptations occur at the level of potentiation and transmission of proprioceptive afferents and thus facilitate sensorimotor activity (movement and balance). Overall, SES is interesting for the improvement/ maintenance of sensorimotor capabilities in non-frail older subjects while NMES is relevant to develop muscle strength/power and thus reduce the risk of falls due to a lack of muscle strength/power in frail older subjects.

Keywords: aging, electrical stimulation, muscle electrical stimulation, somatosensory electrical stimulation, muscle strength, balance, fall, elderly

\section{INTRODUCTION}

Advancing age engenders progressive structural and functional alterations of different organs and systems linked to the motor and postural functions. These alterations naturally and chronologically are likely to lead to motor and postural disturbances related, first, to functional capabilities as part of maximal/intense physical activities, second, to basal functional capabilities as part of activities of daily living, and third, to frailty and an increased risk of falling as part of different body displacements and domestic motor actions - activities at home (ANSES, 2016). A sedentary or inactive life accentuates and accelerates these motor and postural alterations. Although regular physical activity and exercise are the best way of preventing, slowing down 
or limiting these progressive alterations and maintaining the whole functional capabilities (Intiso et al., 2012; Hafström et al., 2016), older subjects are often unable or unwilling to engage in conventional physical activity and exercise or to undertake whole-body physical activity (Paillard, 2018). Hence, in older subjects, in order to limit motor and postural alterations, the optimal (only) solution seems to be the use of artificial techniques, such as the percutaneous peripheral electrical stimulation (Paillard, 2018). This type of stimulation enables the artificial activation of the motor pathway (peripheral and/or central stimulation) and/or the sensory pathway that ensure command, control and execution of movements (Paillard, 2018, 2020, 2021).

However, there are two types of percutaneous peripheral electrical stimulation which, through surface electrodes placed over the bellies or motor points of one (or more) superficial skeletal muscle(s), allow either direct activation of the muscle fibres (i.e., excito-motor stimulation that activates not only the motor and sensory nerve fibres but also the muscle fibres directly) or activation of the sensory nerve fibres only (i.e., sensory stimulation that does not activate muscle fibres and motor nerve fibres; Collins, 2007). Stimulation of sensory nerve fibres can also be applied directly to a nerve - for example, femoral or tibial nerve - or to a joint for example, hip, knee and ankle (Yoshida et al., 2015; Saadat et al., 2017). Excito-motor stimulation is named neuromuscular electrical stimulation (NMES) while sensory stimulation is called sensory (or somatosensory) electrical stimulation (SES). In fact, the type of a stimulation depends on the intensity of the current applied (cf. paragraphs Application modalities and Motor and postural adaptations; Figure 1). Each stimulation induces specific physiological effects on the sensory, central and motor functions related to movement and postural balance with chronic applications (Schröder et al., 2018; Paillard, 2020, 2021). The choice of the stimulation should be based on the specific physiological needs of the older subjects under consideration.

The aim is to propose the type of electrical stimulation best suited to the different physiological profiles of older subjects while specifying the effects induced for each technique and suggesting the underlying mechanistic explanations.

\section{PHYSIOLOGICAL PROFILES AND SPECIFIC RECONDITIONING NEEDS IN OLDER SUBJECTS}

Depending on the physiological profile of older subjects, there are different reconditioning needs for movement and

\section{Neuromuscular electrical stimulation (NMES)}

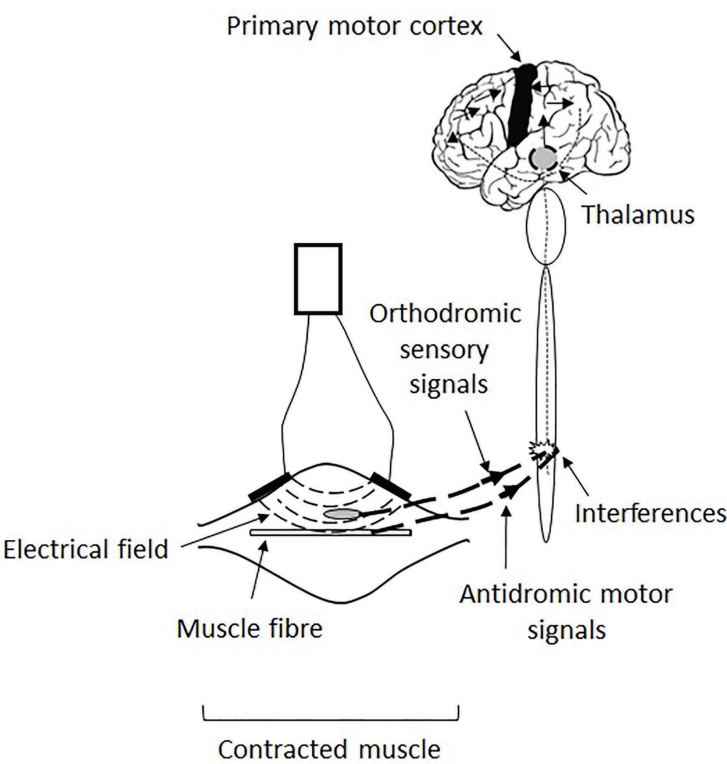

\section{Sensory electrical stimulation}

(SES)

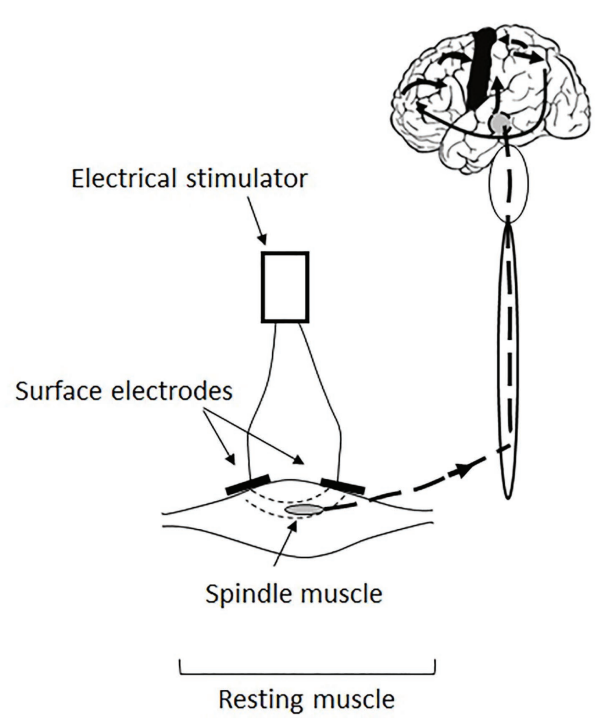

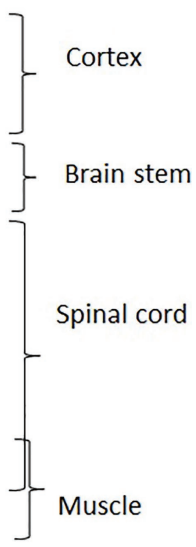

FIGURE 1 | Illustration of the triggering, transmission and central integration of signals generated by neuromuscular electrical stimulation (NMES) and sensory electrical stimulation (SES). NMES involves an excito-motor current, that is, it is strong enough to directly contract the muscle (contracted muscle), whereas SES involves a current below the motor threshold and thus does not induce muscle contraction (resting muscle). In fact, NMES simultaneously activates both sensory and motor neurons and causes a conflict between antidromic motor action potentials and orthodromic sensory action potentials at the spinal level, which interferes with the central integration of the induced afferents (indicated in the diagram of the spinal cord) and thus reduces the ascending sensory volley at the supraspinal level (thin ascending dotted line and thin arrows at cortical level). Hence, NMES generates little exploitable sensory information by the central nervous system to optimise and refine motor and postural skills. In return, SES stimulates only (or almost only) sensory neurons linked to mechanoreceptors which triggers proprioceptive afferents that go up via the thalamus to the postcentral and parietal cortices (primary somatosensory cortex and posterior parietal cortex) to precentral cortices (primary motor cortex) in the stimulated hemisphere (thick ascending dotted line and thick arrows at cortical level). Hence, SES generates enhanced sensorimotor activity that is likely to improve motor and postural capabilities. 
balance. The first physiological profile of advancing age (from a chronological viewpoint) mentioned above (physiological alteration) can be characterised by impaired proprioceptive (myotendinous and articular cues), vestibular (otolithic and semicircular cues) and exteroceptive (visual and skin cues) input and/or integration and/or decreased motor output (Maitre et al., 2013). With this physiological profile, the needs are mainly sensorimotor. Moreover, since the absence of physical activity accentuates all the impairments mentioned above, the functional capabilities are primarily and clearly impeded due to excessive degradation of motor output (Paillard, 2017b; Trajkov et al., 2018). This corresponds to the second physiological profile described above and its needs are primarily based on the development of muscle strength/ power (pure motor output) at least in order to partially recondition the basal functional capabilities more easily (Paillard, 2017b). For the third physiological profile corresponding to an increased fall risk, evidence suggests that the needs are the same but are still much more pronounced than those in the second physiological profile related to the absolute needs to develop muscle strength/power (Paillard, 2017b). Whether it is a question of developing sensorimotor function or only motor function (e.g., muscle strength/power), the technique of electrical stimulation constitutes an excellent means for reconditioning older subjects corresponding to the second and third physiological profiles, but it can also be appropriately used with subjects of the first physiological profile, especially when they are sedentary or inactive. However, the type of electrical stimulation required depends on whether the aim is to develop sensorimotor function or only motor function.

\section{MOTOR AND POSTURAL ADAPTATIONS INDUCED BY ELECTRICAL STIMULATION}

\section{Neuromuscular Electrical Stimulation Application Modalities}

NMES is excito-motor which means that it directly activates muscle fibres by bypassing motor neurons (even if they are simultaneously activated as well as sensory neurons). To this end, it must be clearly above the motor threshold, that is, the minimal intensity of stimulation that produces a direct motor response and generates involuntary non-controlled segmental movements. It turns out that the higher the intensity, the greater the extent of the electrical field and the greater the number of recruited muscle fibres - that is, both I and II fibre types (Collins, 2007). In the context of regular or chronic application, it appears that the higher the current intensity, the greater the physiological effects/benefits induced (Paillard, 2018). NMES produces strong muscle contractions and provokes pain through surface electrodes placed over the bellies or motor points of muscle(s) targeted (Lake, 1992; Vanderthommen and Duchateau, 2007). In order to recondition the basal functional capabilities in older (frail) subjects, NMES should be applied specifically to the muscles of their lower limbs with high intensities (maximal tolerable by subject), high frequencies $(>30 \mathrm{~Hz}$ and rather $50-80 \mathrm{~Hz}$ ), optimal width pulses (matching to the chronaxy of the stimulated muscle that is, for instance $300-450 \mu \mathrm{s}$ for quadriceps femoris) short contractions interspersed with long recovery times - for example, 3-10 s/10-30s on/off - for $10-15 \mathrm{~min}, 20 \mathrm{~min}$ maximum (Paillard, 2018).

\section{Motor and Postural Adaptations}

Evidence suggests that NMES regularly applied on quadriceps femoris or dorsi/plantarflexor muscles (e.g., tibialis anterior, and soleus) in older subjects ( $>60$ years old) improves lowerlimb muscle strength (Caggiano et al., 1994; Paillard et al., 2004; Bezerra et al., 2011; Caulfield et al., 2013; Kern et al., 2014; Mignardot et al., 2015; Von Stengel et al., 2015; Mani et al., 2018; Acaröz Candan et al., 2019; Langeard et al., 2021) and postural balance (Amiridis et al., 2005; Paillard et al., 2005a,b; Nejc et al., 2013; Mignardot et al., 2015; Alptekin et al., 2016; Bondi et al., 2021).

In this context, the functional improvements of motor and postural functions in older subjects are mainly linked to enhancements of motor output through muscle structural (mass) and functional (neural networks) adaptations - Figure 2 (Paillard, 2018). The naturally irreversible atrophy of lowerlimb muscle in inactive or sedentary individuals can be reversed with the chronic application of NMES (Boncompagni et al., 2007; Carraro et al., 2015). NMES stimulates not only anabolic pathways (e.g., secretion of insulin-like growth factor-1), but also negatively modulates muscle catabolism, which increases protein synthesis and reduces protein degradation and activates satellite cells in aged individuals (Barberi et al., 2015; Mancinelli et al., 2019). Hence, NMES induces an increase in the size of muscle fibres especially in the type II muscle fibres which are particularly affected by the effects of advancing age (Zampieri et al., 2015; Mancinelli et al., 2019). From a functional viewpoint, NMES results in greater contribution of muscles regularly stimulated (i.e., electromyographic activity of tibialis anterior and medial gastrocnemius) in the postural regulation (Amiridis et al., 2005). It also entails increased musculotendinous stiffness of the muscles regularly stimulated (Mignardot et al., 2015).

However, even if one cannot ignore that NMES applied on selected muscle groups engenders sensory information likely to be used as part of the command and control of voluntary movement - for example, during walking - Paillard (2020) inferred that it generates little sensory information likely to be exploited by the central nervous system to optimise and refine perceptual and motor skills as part of the postural balance regulation (Figure 2). In fact, NMES simultaneously activates both sensory and motor neurons and would provoke conflict between the antidromic motor action potentials and the orthodromic sensory action potentials at the spinal level (Bergquist et al., 2011), thus causing interference in the central integration of induced afferents (although the presence of central adaptations cannot be totally excluded). 


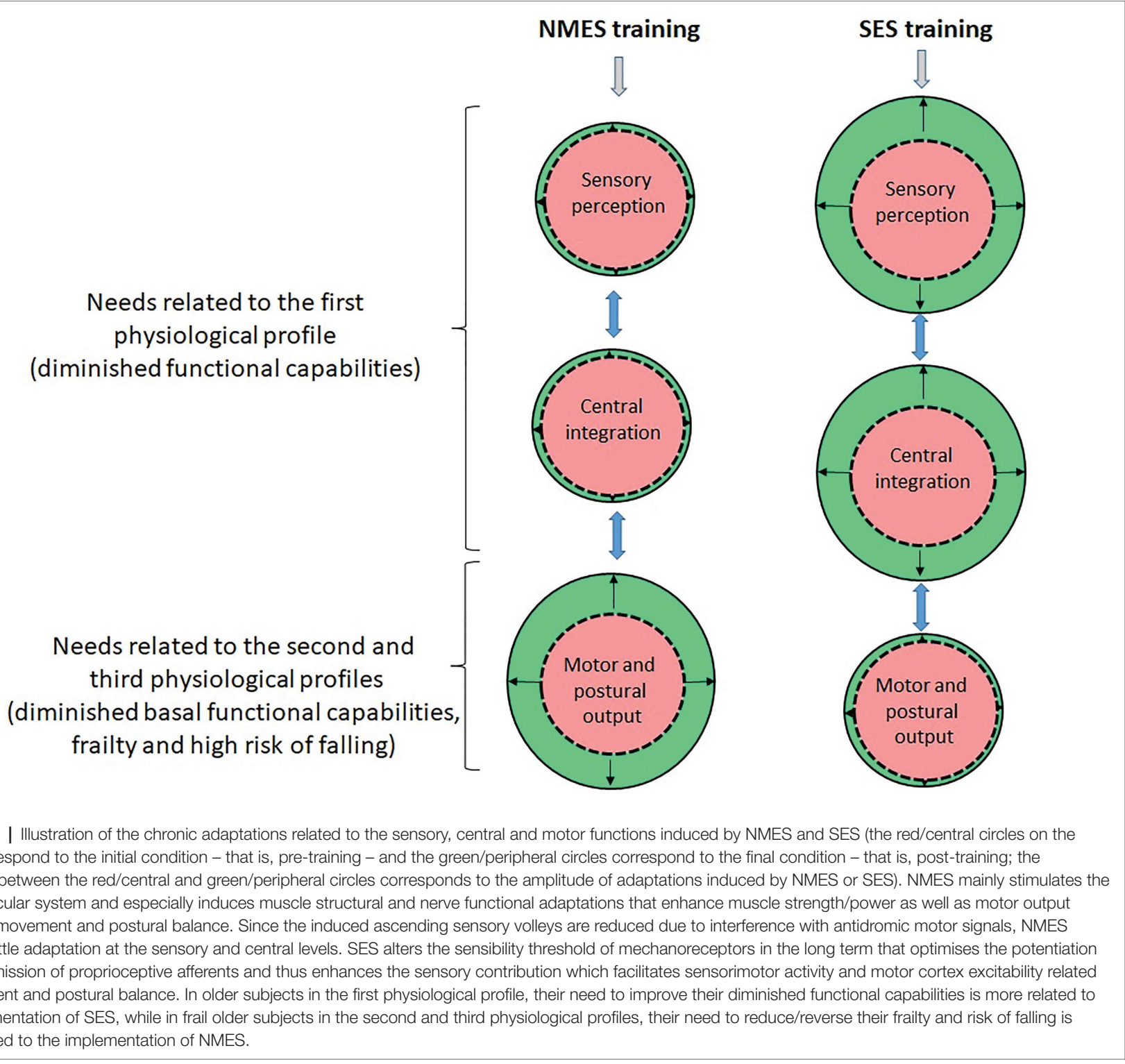

In order to improve the functional capabilities, NMES would be particularly interesting since it is likely to enhance motor output especially muscle strength/power in older frail subjects with a high risk of falling precisely due to a lack of muscle strength/power.

\section{Sensory Electrical Stimulation Application Modalities}

SES is not excito-motor (it cannot directly activate the muscle fibres) and only (or almost only) activates the sensory pathways (this is the principle of this stimulation). To this end, it must be well below the motor threshold and close to the sensory threshold, that is, the minimum intensity of stimulation that can be perceived by individual. Indeed, the current intensity must be around the sensory threshold without exceeding it or only slightly, since a non-excito-motor current (low intensity) is already likely to lead to recruitment of type I muscle fibres from reflex pathways - mediated at spinal level, that is, homolateral monosynaptic connections of the Ia fibres with the $\alpha$-motoneurons generate depolarisation of the latter and induce the contraction of the muscle fibres they innervate (Zeronian et al., 2021). SES is painless (although there are possible sensations) and applied to a peripheral nerve, belly muscles (motor points) and/or joints at current intensities below, at or slightly above the sensory threshold (Schröder et al., 2018; Paillard, 2021). In order to recondition the motor and postural functions in older subjects, SES should be applied with large pulses (e.g., $1 \mathrm{~ms})$ and high frequencies $(80-100 \mathrm{~Hz})$ to facilitate potentiation and central integration of emitted signals (Collins, 2007; Bergquist et al., 2011) for several tens of minutes in a uninterrupted way.

\section{Motor and Postural Adaptations}

Evidence suggests that SES regularly applied to lower-limb muscle (motor points), peripheral nerve or joints in older subjects 
( $>60$ years old) improves sensorimotor function (Park et al., 2014; Ng et al., 2016; Paillard, 2021). However, since SES generates no muscle deformation or contraction, it thus cannot stimulate synthesis of contractile proteins responsible for muscle structural adaptations (Coffey and Hawley, 2007). The adaptations induced cannot occur at the level of the motor output of muscle. In fact, SES generates sensory cues that are detected by sensory sensors which transmit signals throughout sensory pathways to cortical areas (Figure 1). With chronic application, SES induces adaptations at different stages (sites) throughout sensory pathways - sensory sensors and spinal and supraspinal structures (Figure 2).

At the peripheral level (i.e., sensory sensors), SES would modify the sensitivity threshold of mechanoreceptors that result from altering the ion permeability of their membrane (Gravelle et al., 2002; Ross et al., 2007). This sensory adaptation would optimise the potentiation and transmission of proprioceptive afferents and thus enhance the sensory contribution in the motor and postural regulation (Ross et al., 2007; Severini and Delahunt, 2018; Paillard, 2021). At the spinal level, SES is likely to engender a reduction or increase in the amplitude of the induced $\mathrm{H}$-reflex (Goulet et al., 1997; Hardy et al., 2002, respectively) that, in both cases, can improve motor and postural abilities (Paillard, 2017a). The reduction of the Ia-afferent excitation to the $\alpha$-motoneuron pool can attenuate destabilising joint movements while the enhancement of the activation of the $\alpha$-motoneuron pool can facilitate instantaneous segmental reactions to the demands of the motor or postural task (Paillard, 2017a). At the cortical level, SES is likely to induce durable changes in motor cortex excitability that can be assimilated to enhance sensorimotor activity and connectivity (Kaelin-Lang et al., 2002; Schröder et al., 2018; Insausti-Delgado et al., 2021). Enhanced sensorimotor activity could result from favourable impact of SES over postcentral and parietal cortices (primary somatosensory cortex and posterior parietal cortex) to precentral cortices (primary motor cortex) in the stimulated hemisphere (Paillard, 2021).

SES would be of particular interest as it is likely to improve sensorimotor output in older subjects who are not at high risk of falling due to lack of muscle strength/power or very weak motor output.

\section{REFERENCES}

Acaröz Candan, S., Akoğlu, A. S., Büğüşan, S., and Yüksel, F. (2019). Effects of neuromuscular electrical stimulation of quadriceps on the quadriceps strength and functional performance in nursing home residents: a comparison of short and long stimulation periods. Geriatr Gerontol Int 19, 409-413. doi: $10.1111 /$ ggi.13633

Alptekin, K., Karan, A., Diracoglu, D., Yildiz, A., Baskent, A., and Eskiyurt, N. (2016). Investigating the effectiveness of postural muscle electrostimulation and static posturography feedback exercises in elders with balance disorder. J. Back Musculoskelet. Rehabil. 29, 151-159. doi: 10.3233/ BMR-150611

Amiridis, I., Arabatzi, F., Violaris, P., Stavropoulos, E., and Hatzitaki, V. (2005). Static balance improvement in elderly after dorsiflexors electrostimulation training. Eur. J. Appl. Physiol. 94, 424-433. doi: 10.1007/ s00421-005-1326-3

ANSES (2016). Actualisation des repères du PNNS - Révisions des repères relatifs à l'activité physique et à la sédentarité/Collective expertise - National health nutrition program and revisions of benchmarks relating to physical

\section{WHICH ELECTRICAL STIMULATION FOR WHICH PHYSIOLOGICAL PROFILE IN OLDER SUBJECTS?}

On the basis of an older subject's needs, belonging to the first physiological profile, SES would turn out to be particularly interesting since it especially facilitates the sensorimotor reconditioning and thus optimises functional capabilities (Figure 2). For older subjects in the second physiological profile who exhibit diminished basal functional capabilities, NMES would turn out to be relevant since it can initiate/trigger structural and functional muscle adaptations that can reverse the process of reduction of functional capabilities in activities of daily living (Figure 2). Regarding older subjects in the third physiological profile who are at high risk of falling due to extreme frailty and a notable lack of muscle strength/power in the lower-limb, NMES would reverse the process of frailty and muscle involution, thus reducing the risk of falling.

\section{CONCLUSION}

In older subjects, as long as their basal functional capabilities are not clearly limited/reduced as part of activities of daily living and their risk of falling is low, SES is particularly useful for maintaining or even improving their capabilities to command and control movement and postural balance. In turn, in frail older subjects with diminished basal functional capabilities and at high risk of falling, NMES can potentially boost their neuromuscular system in order to recondition the lower-limb muscle strength/power thus limiting their risk of falling.

\section{AUTHOR CONTRIBUTIONS}

The author confirms being the sole contributor of this work and has approved it for publication.

activity and sedentarity Agence Nationale de Sécurité Sanitaire Alimentation, Environnement Travail. Paris, 584.

Barberi, L., Scicchitano, B. M., and Musaro, A. (2015). Molecular and cellular mechanisms of muscle aging and sarcopenia and effects of electrical stimulation in seniors. Eur. J. Trans. Myol. Basic Appl. Myol. 25, 231-236. doi: 10.4081/ejtm.2015.5227

Bergquist, A. J., $\quad$ Clair, J. M., $\quad$ Lagerquist, O., $\quad$ Mang, C. S., $\quad$ Okuma, Y., and Collins, D. F. (2011). Neuromuscular electrical stimulation: implications of the electrically evoked sensory volley. Eur. J. Appl. Physiol. 111, 2409-2426. doi: 10.1007/s00421-011-2087-9

Bezerra, P., Zhou, S., Crowley, Z., Davie, A., and Baglin, R. (2011). Effects of electromyostimulation on knee extensors and flexors strength and steadiness in older adults. J. Mot. Behav. 43, 413-421. doi: 10.1080/00222895.2011.620039

Boncompagni, S., Kern, H., Rossini, K., Hofer, C., Mayr, W., Carraro, U., et al. (2007). Structural differentiation of skeletal muscle fibers in the absence of innervation in humans. Proc. Natl. Acad. Sci. U. S. A. 104, 19339-19344. doi: 10.1073/pnas.0709061104

Bondi, D., Tereza, J., Verratti, V., D’Amico, M., Kinel, E., D’Attilio, M., et al. (2021). Static balance adaptations after neuromuscular electrical 
stimulation on quadriceps and lumbar paraspinal muscles in healthy elderly. Sport Sci. Health. doi: 10.1007/s11332-021-00777-0

Caggiano, E., Emrey, T., Shirley, S., and Craik, R. L. (1994). Effects of electrical stimulation or voluntary contraction for strengthening the quadriceps femoris muscles in an aged male population. J. Orthop. Sports Phys. Ther. 20, 22-28. doi: 10.2519/jospt.1994.20.1.22

Carraro, U., Kern, H., Gava, P., Hofer, C., Loefler, S., Gargiulo, P., et al. (2015). Biology of muscle atrophy and of its recovery by FES in aging and mobility impairments: roots and by-products. Eur. J. Transl. Myol. 25, 221-230. doi: 10.4081/ejtm.2015.5272

Caulfield, B., Prendergast, A., Rainsford, G., and Minogue, C. (2013). Self-directed home based electrical muscle stimulation training improves exercise tolerance and strength in healthy elderly. Annu. Int. Conf. IEEE Eng. Med. Biol. Soc. 2013, 7036-7039. doi: 10.1109/ EMBC.2013.6611178

Coffey, V. G., and Hawley, J. A. (2007). The molecular bases of training adaptation. Sports Med. 37, 737-763. doi: 10.2165/00007256-20073709000001

Collins, D. F. (2007). Central contributions to contractions evoked by tetanic neuromuscular electrical stimulation. Exerc. Sport Sci. Rev. 35, 102-109. doi: 10.1097/jes.0b013e3180a0321b

Goulet, C. G., Arsenault, A. B., Bourbonnais, D., and Levin, M. F. (1997). Effects of transcutaneous electrical nerve stimulation on the H-reflex of muscles of different fibre type composition. Electromyogr. Clin. Neurophysiol. $37,335-342$.

Gravelle, D. C., Laughton, C. A., Dhruv, N. T., Katdare, K. D., Niemi, J. B., Lipsitz, L. A., et al. (2002). Noise-enhanced balance control in older adults. Neuroreport 13, 1853-1856. doi: 10.1097/00001756-20021028000004

Hafström, A., Malmström, E. M., Terdèn, J., Fransson, P. A., and Magnusson, M. (2016). Improved balance confidence and stability for elderly after 6 weeks of a multimodal self-administered balance-enhancing exercise program: a randomized single arm crossover study. Gerontol. Geriatr. Med. 2:233372141664414. doi: 10.1177/2333721416644149

Hardy, S. G., Spalding, T. B., Liu, H., Nick, T. G., Pearson, R. H., Hayes, A. V., et al. (2002). The effect of transcutaneous electrical stimulation on spinal motor neuron excitability in people without known neuromuscular diseases: the roles of stimulus intensity and location. Phys. Ther. 82, 354-363. doi: $10.1093 / \mathrm{ptj} / 82.4 .354$

Insausti-Delgado, A., López-Larraz, E., Omedes, J., and Ramos-Murguialday, A. (2021). Intensity and dose of neuromuscular electrical stimulation influence sensorimotor cortical excitability. Front. Neurosci. 14:593360. doi: 10.3389/ fnins. 2020.593360

Intiso, D., Di Rienzo, F., Russo, M., Pazienza, L., Tolfa, M., Larossi, A., et al. (2012). Rehabilitation strategy in the elderly. J. Nephrol. 25(Suppl. 19), S90-S95. doi: 10.5301/jn.5000138

Kaelin-Lang, A., Luft, A. R., Sawaki, L., Burstein, A. H., Sohn, Y. H., and Cohen, L. G. (2002). Modulation of human corticomotor excitability by somatosensory input. J. Physiol. 540, 623-633. doi: 10.1113/ jphysiol.2001.012801

Kern, H., Barberi, L., Löfler, S., Sbardella, S., Burggraf, S., Fruhmann, H., et al. (2014). Electrical stimulation counteracts muscle decline in seniors. Front. Aging Neurosci. 6:189. doi: 10.3389/fnagi.2014.00189

Lake, D. A. (1992). Neuromuscular electrical stimulation. An overview and its application in the treatment of sports injuries. Sports Med. 13, 320-336. doi: 10.2165/00007256-199213050-00003

Langeard, A., Bigot, L., Loggia, G., Bherer, L., Chastan, N., and Gauthier, A. (2021). Ankle dorsiflexors and plantarflexors neuromuscular electrical stimulation training impacts gait kinematics in older adults: a pilot study. Gait Posture 84, 335-339. doi: 10.1016/j. gaitpost.2020.12.016

Maitre, J., Gasnier, Y., Bru, N., Jully, J. L., and Paillard, T. (2013). Discrepancy in the involution of the different neural loops with age. Eur. J. Appl. Physiol. 113, 1821-1831. doi: 10.1007/s00421-013-2608-9

Mancinelli, R., Toniolo, L., Di Filippo, E. S., Doria, C., Marrone, M., Maroni, C. R., et al. (2019). Neuromuscular electrical stimulation induces skeletal muscle fiber remodeling and specific gene expression profile in healthy elderly. Front. Physiol. 10:1459. doi: 10.3389/ fphys.2019.01459
Mani, D., Almuklass, A. M., Amiridis, I. G., and Enoka, R. M. (2018). Neuromuscular electrical stimulation can improve mobility in older adults but the time course varies across tasks: double-blind, randomized trial. Exp. Gerontol. 108, 269-275. doi: 10.1016/j.exger.2018. 04.018

Mignardot, J. B., Deschamps, T., Le Goff, C. G., Roumier, F. X., Duclay, J., Martin, A., et al. (2015). Neuromuscular electrical stimulation leads to physiological gains enhancing postural balance in the pre-frail elderly. Phys. Rep. 3:e12471. doi: 10.14814/phy2.12471

Nejc, S., Loefler, S., Cvecka, J., Sedliak, M., and Kern, H. (2013). Strength training in elderly people improves static balance: a randomized controlled trial. Eur. J. Transl. Myol Basic Appl. Myol. 23, 85-89. doi: 10.4081/ bam.2013.3.85

Ng, S. S., Lai, C. W., Tang, M. W., and Woo, J. (2016). Cutaneous electrical stimulation to improve balance performance in patients with subacute stroke: a randomised controlled trial. Hong Kong Med. J. 22(Suppl. 2), 33-36.

Paillard, T. (2017a). Plasticity of the postural function to sport and/or motor experience. Neurosci. Biobehav. Rev. 72, 129-152. doi: 10.1016/j. neubiorev.2016.11.015

Paillard, T. (2017b). Relationship between muscle function, muscle typology and postural performance according to different postural conditions in young and older adults. Front. Physiol. 8:585. doi: 10.3389/fphys.2017.00585

Paillard, T. (2018). Muscle plasticity of aged subjects in response to electrical stimulation training and inversion and/or limitation of the sarcopenic process. Ageing Res. Rev. 46, 1-13. doi: 10.1016/j.arr.2018.05.002

Paillard, T. (2020). Acute and chronic neuromuscular electrical stimulation and postural balance: a review. Eur. J. Appl. Physiol. 120, 1475-1488. doi: 10.1007/s00421-020-04383-9

Paillard, T. (2021). Sensory electrical stimulation and postural balance: a comprehensive review. Eur. J. Appl. Physiol. 121, 3261-3281. doi: 10.1007/ s00421-021-04767-5

Paillard, T., Lafont, C., Pérès, C., Costes-salon, M. C., Soulat, J. M., Montoya, R., et al. (2005a). Is electrical stimulation with voluntary muscle contraction exercice? Of physiologic interest in aging women? Ann. Readapt. Med. Phys. 48, 20-28. doi: 10.1016/j.annrmp.2004. 08.005

Paillard, T., Lafont, C., Soulat, J. M., Costes-Salon, M. C., Mario, B., Montoya, R., et al. (2004). Neuromuscular effects of three training methods in ageing women. J. Sports Med. Phys. Fitness 44, 87-91.

Paillard, T., Lafont, C., Soulat, J. M., Montoya, R., Costes-Salon, M. C., and Dupui, P. (2005b). Short-term effects of electrical stimulation superimposed on muscular voluntary contraction in postural control in elderly women. J. Strength Cond. Res. 19, 640-646. doi: 10.1519/ 00124278-200508000-00026

Park, J., Seo, D., Choi, W. , and Lee, S. (2014). The effects of exercise with TENS on spasticity, balance, and gait in patients with chronic stroke: a randomized controlled trial. Med. Sci. Monit. 20, 1890-1896. doi: 10.12659/ MSM.890926

Ross, S. E., Arnold, B. L., Blackburn, J. T., Brown, C. N., and Guskiewicz, K. M. (2007). Enhanced balance associated with coordination training with stochastic resonance stimulation in subjects with functional ankle instability: an experimental trial. J. Neuroeng. Rehabil. 4:47. doi: 10.1186/1743-0003-4-47

Saadat, Z., Rojhani-Shirazi, Z., and Abbasi, L. (2017). Dose postural control improve following application of transcutaneous electrical nerve stimulation in diabetic peripheral neuropathic patients? A randomized placebo control trial. Diabetes Metab. Syndr. 11, S755-S757. doi: 10.1016/j. dsx.2017.05.011

Schröder, J., Truijen, S., Van Criekinge, T., and Saeys, W. (2018). Peripheral somatosensory stimulation and postural recovery after stroke a systematic review. Top. Stroke Rehabil. 25, 312-320. doi: 10.1080/10749357.2018.1440694

Severini, G., and Delahunt, E. (2018). Effect of noise stimulation below and above sensory threshold on postural sway during a mildly challenging balance task. Gait Posture 63, 27-32. doi: 10.1016/j.gaitpost.2018.04.031

Trajkov, M., Cuk, I., Eminovic, F., Kljajic, D., and Dopsaj, M. (2018). Relationship between hand grip strength and endurance and postural stability 
in active and sedentary older women. J. Electromyogr. Kinesiol. 43, 62-67. doi: 10.1016/j.jelekin.2018.09.006

Vanderthommen, M., and Duchateau, J. (2007). Electrical stimulation as a modality to improve performance of the neuromuscular system. Exerc. Sport Sci. Rev. 35, 180-185. doi: 10.1097/jes.0b013e318156e785

Von Stengel, S., Bebenek, M., Engelke, K., and Kemmler, W. (2015). Whole-body electromyostimulation to fight osteopenia in elderly females: the randomized controlled training and electrostimulation trial (TEST-III). J. Osteoporos. 2015:643520. doi: 10.1155/2015/643520

Yoshida, T., Tanino, Y., and Suzuki, T. (2015). Effect of exercise therapy combining electrical therapy and balance training on functional instability resulting from ankle sprain-focus on stability of jump landing. J. Phys. Ther. Sci. 27, 3069-3071. doi: 10.1589/jpts.27.3069

Zampieri, S., Mosole, S., Löfler, S., Fruhmann, H., Burggraf, S., Cvečka, J., et al. (2015). Physical exercise in aging: nine weeks of leg press or electrical stimulation training in 70 years old sedentary elderly people. Eur. J. Transl. Myol. 25, 237-242. doi: 10.4081/ejtm.2015.5374

Zeronian, S., Noe, F., and Paillard, T. (2021). Effect of the application of somatosensory and excitomotor electrical stimulation during quiet upright standing balance. Med. Eng. Phys. 87, 82-86. doi: 10.1016/j.medengphy. 2020.11.016

Conflict of Interest: The author declares that the research was conducted in the absence of any commercial or financial relationships that could be construed as a potential conflict of interest.

Publisher's Note: All claims expressed in this article are solely those of the authors and do not necessarily represent those of their affiliated organizations, or those of the publisher, the editors and the reviewers. Any product that may be evaluated in this article, or claim that may be made by its manufacturer, is not guaranteed or endorsed by the publisher.

Copyright (๑) 2022 Paillard. This is an open-access article distributed under the terms of the Creative Commons Attribution License (CC BY). The use, distribution or reproduction in other forums is permitted, provided the original author(s) and the copyright owner(s) are credited and that the original publication in this journal is cited, in accordance with accepted academic practice. No use, distribution or reproduction is permitted which does not comply with these terms. 